\title{
ORIGINAL
}

\section{Comparative study of lung ultrasound and chest computed tomography scan in the assessment of severity of confirmed COVID-19 pneumonia}

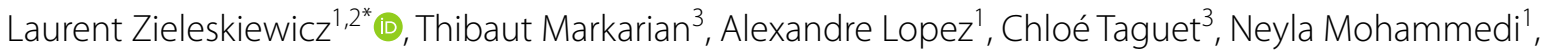 \\ Mohamed Boucekine ${ }^{4}$, Karine Baumstarck ${ }^{4}$, Guillaume Besch ${ }^{5}$, Gautier Mathon ${ }^{6}$, Gary Duclos ${ }^{1}$, \\ Lionel Bouvet ${ }^{7,8,9}$, Pierre Michelet ${ }^{3}$, Bernard Allaouchiche ${ }^{6,8,9}$, Kathia Chaumoître $^{10}$, Mathieu Di Bisceglie ${ }^{10}$ and \\ Marc Leone ${ }^{1}$ on behalf of the AZUREA Network
}

(c) 2020 Springer-Verlag GmbH Germany, part of Springer Nature

\begin{abstract}
Purpose: The relationship between lung ultrasound (LUS) and chest computed tomography (CT) scans in patients with severe acute respiratory syndrome coronavirus 2 (SARS-CoV-2) pneumonia is not clearly defined. The primary objective of our study was to assess the performance of LUS in determining severity of SARS-CoV-2 pneumonia compared with chest CT scan. Secondary objectives were to test the association between LUS score and location of the patient, use of mechanical ventilation, and the pulse oximetry $\left(\mathrm{SpO}_{2}\right) /$ fractional inspired oxygen $\left(\mathrm{FiO}_{2}\right)$ ratio.

Methods: A multicentre observational study was performed between 15 March and 20 April 2020. Patients in the Emergency Department (ED) or Intensive Care Unit (ICU) with acute dyspnoea who were PCR positive for SARS-CoV-2, and who had LUS and chest CT performed within a 24-h period, were included.

Results: One hundred patients were included. LUS score was significantly associated with pneumonia severity assessed by chest CT and clinical features. The AUC of the ROC curve of the relationship of LUS versus chest CT for the assessment of severe SARS-CoV-2 pneumonia was 0.78 (CI 95\% 0.68-0.87; $p<0.0001)$. A high LUS score was associated with the use of mechanical ventilation, and with a $\mathrm{SpO}_{2} / \mathrm{FiO}_{2}$ ratio below 357 .

Conclusion: In known SARS-COV-2 pneumonia patients, the LUS score was predictive of pneumonia severity as assessed by a chest CT scan and clinical features. Within the limitations inherent to our study design, LUS can be used to assess SARS-CoV-2 pneumonia severity.
\end{abstract}

Keywords: SARS-CoV-2, Chest computed tomography, Lung ultrasound, Diagnostic accuracy

*Correspondence: laurent.zieleskiewicz@ap-hm.fr

1 Department of Anesthesiology and Intensive Care Medicine, Hôpital Nord, Assistance Publique Hôpitaux de Marseille, Aix Marseille University, 13015 Marseille, France

Full author information is available at the end of the article

Laurent Zieleskiewicz and Thibaut Markarian are joint first authors and contributed equally.

\section{Springer}

\section{Introduction}

Severe acute respiratory syndrome from coronavirus 2 (SARS-CoV-2) can be identified by a chest computed tomography $(\mathrm{CT})$ scan $[1,2]$, typically showing ground-glass opacities, consolidation and interlobular septal thickening [3]. CT scanning has recently been shown to predict prognosis in these patients [4]. However, the use of chest CT scan is associated with adverse events and increased resource consumption $[5,6]$, and especially during this pandemic, the safety 
of transferring patients for CT scan remains uncertain at both individual and collective levels [7].

Lung ultrasound (LUS) has a high diagnostic accuracy for interstitial syndrome and alveolar consolidation, which is superior to chest radiograph $[8,9]$. LUS has been recommended for the diagnosis and management of pneumonia [10], even during a previous viral pandemic [11]. During the current coronavirus disease 2019 (COVID-19) pandemic, LUS might reduce inhospital transfers, the exposure of healthcare workers, and the risk of contamination of medical devices [12].

The main pattern of SARS-CoV-2 pneumonia using LUS is interstitial syndrome [13]. However, pneumonia may show different features using different diagnostic methods $[3,14]$. To date, there has been case report [15], but few studies, comparing LUS with chest CT scan in patients with SARS-CoV-2 pneumonia.

The primary objective of our study was to assess the performance of LUS in determining COVID-19 pneumonia severity as assessed by chest CT scan. The secondary objectives were to test the association between LUS score and clinical features including the location of the patient, mechanical ventilation and pulse oximetry $\left(\mathrm{SpO}_{2}\right) /$ fractional inspired oxygen $\left(\mathrm{FiO}_{2}\right)$ ratio.

\section{Methods \\ Design}

This multicentre observational study was conducted in four university hospitals. We performed a retrospective analysis comparing the result of LUS examinations and chest $\mathrm{CT}$, performed as part of routine care, in a convenience sample of patients in either the Emergency Department (ED) or the Intensive care unit (ICU). The ED and ICUs were located in different institutions.

\section{Ethical considerations}

The study was approved by the Committee for Research Ethics of the French Society of Anesthesia and Intensive Care Medicine (CERAR IRB000102542020-062). In accordance with French law, patients were informed regarding the use of their data for publication [16].

\section{Population}

We included adult patients admitted to the ED or ICU with confirmed SARS-CoV-2 infection, diagnosed by acute dyspnoea $\left(\mathrm{SpO}_{2}<94 \%\right.$ and/or breathlessness [17]) together with a positive polymerase chain reaction (PCR) test in a nasopharyngeal or bronchoalveolar sample, who had a LUS exam at admission as well as a chest $\mathrm{CT}$ within the $24 \mathrm{~h}$ following the LUS.

\section{Take-home message}

Lung ultrasound is a possible alternative to chest CT scan, especially in resource-constrained environment, for the diagnosis of SARS-

CoV-2 pneumonia severity. Given its high availability at the bedside, it may be widely used during the pandemic

\section{Clinical features}

At inclusion, each patient had a standard medical history and examination, monitoring of heart and respiratory rate, blood pressure and $\mathrm{SpO}_{2}$, and arterial blood gas analysis.

For patients who were breathing spontaneously, $\mathrm{FiO}_{2}$ was calculated as follows: $\mathrm{FiO}_{2}=(21+3 *$ oxygen flow $(\mathrm{L} /$ $\min ) / 100)$ [18].

For the purposes of analysis, we defined a low $\mathrm{SpO}_{2} /$ $\mathrm{FiO}_{2}$ ratio as $<357$ and high $\mathrm{SpO} 2 / \mathrm{FiO} 2$ ratio as $\geq 357$. This cut-off was chosen as it is equivalent to a $\mathrm{PaO}_{2} / \mathrm{FiO}_{2}$ ratio of $300 \mathrm{mmHg}$ [19].

\section{LUS and chest CT examination}

LUS was performed within the first $2 \mathrm{~h}$ after admission. LUS was performed by imaging 12 lung regions, modified for critical illness. Thus, we imaged the posterior areas behind the posterior axillary line rather than in the paravertebral areas to avoid turning completely the patient [20]. LUS examinations were performed by emergency physicians or intensivists in charge of the patient [21,22]. The skill of the operators was rated as follows [21]:

- Level 3 operator; LUS academic teacher with several publications in the field

- Level 2 operator; more than 25 supervised procedures and 200 non-supervised procedures

- Level 1 operator; at least 25 supervised procedures and less than 200 non-supervised procedures

Chest CT was performed at an appropriate time during the clinical course using a 128-slice CT (OPTIMA CT660, GE Healthcare, Chicago, Illinois, US) in the supine position, with the patient instructed to hold their breath after a deep inspiration. Most CT scans were noncontrast, low-dose chest CT.

Further details of the diagnostic procedures are available in the Electronic Supplemental Material (ESM 1).

\section{Sample size considerations}

The sample size calculation was performed to determine whether an area under the curve (AUC) of $\geq 0.80$ was achieved for a receiver operator characteristic (ROC) plot of LUS versus chest CT scan. Based on the unpublished data, with a precision of $10 \%$ and an expected proportion 
of severe pneumonia on chest CT scan of $40 \%$, the sample size required was 87 . Taking into account the potential for incomplete data from LUS or chest CT scans, we included 100 patients.

\section{Statistical analysis}

The characteristics of the patients are summarized as medians and interquartile ranges for continuous variables, and as numbers and percentages for qualitative variables. Comparisons of patients' characteristics, CT and ultrasound parameters were performed between patients managed in the ED versus the ICU. The LUS score was compared between the three severity grades (mild, moderate, and severe pneumonia) on CT scan using an ANOVA test. The receiver operator characteristic (ROC) curve and AUC estimates were determined for the relationship of LUS score and CT scan to diagnose severity of pneumonia. The optimal threshold for best discrimination between non-severe and severe pneumonia was calculated using the Youden index. Sensitivity (Se), specificity (Sp), negative predictive value (NPV), positive predictive value (PPV) and DA are provided with their 95\% confidence intervals (CIs). A grey zone represents a predictive test of low accuracy, that is, the Se and $\mathrm{Sp}$ are both $<90 \%$ [23]. Se and Sp curves were constructed to calculate the grey zone for an LUS score that was inconclusive for predicting severe pneumonia [24]. ROC and AUC, the optimal thresholds, Se, Sp, NPV, PPV and DA were determined for LUS score to diagnose consolidation, interstitial syndrome, pleural effusion and pleural irregularity according to the chest $\mathrm{CT}$ findings and use of mechanical ventilation. Mean LUS scores were compared between low and high $\mathrm{SpO}_{2} / \mathrm{FiO}_{2}$ ratios. For all calculations, $\mathrm{R}$ software (R Development Core Team) and SPSS Statistics for Windows, Version 20.0 (IBM, Armonk, NY) were used. The significance level was set at $p<0.05$.

\section{Results}

\section{Patient features}

Figure 1 presents the flowchart of the study. Among the 412 patients presenting with suspected SARS-CoV-2 infection in the ED, 207 were symptomatic with dyspnea. Of these symptomatic patients, 77 underwent LUS exam followed by a chest CT scan within the next $24 \mathrm{~h}$ and were included in the ED group. Among the 87 patients admitted to the ICU during this period, 23 fulfilled the inclusion criteria and were included in the ICU group. Thus, 100 patients (200 hemi-thoraces) were analyzed. The demographic and clinical data of the patients are summarized in Table 1.

\section{Descriptive analysis}

LUS was performed by Level 3, Level 2 and Level 1 operators in $37 \%, 53 \%$ and $10 \%$ of cases, respectively. A descriptive analysis of chest CT scan and LUS findings is reported in (Table 2).

\section{Primary outcome}

The LUS score was significantly associated with chest CT scan severity $(p<0.0001$; Table 3$)$. The AUC of the ROC curve of the relationship of LUS versus chest CT for the assessment of severe SARS-CoV-2 pneumonia was 0.78 (CI 95\% 0.68-0.87; $p<0.0001$; Fig. 2a). The maximum Youden index was 0.50 . The LUS score gray zone (score of 13-23) with inconclusive values included 38 patients (38\%) (Fig. 2b). An LUS score $>23$ predicted severe SARS-CoV-2 pneumonia diagnosed by chest CT scan with a $\mathrm{Sp}>90 \%$ and a PPV of $70 \%$ in 23 patients. An LUS score $<13$ excluded severe SARS-CoV-2 pneumonia diagnosed by chest CT scan with a Se $>90 \%$ and an NPV of $92 \%$ in 39 patients. Thirty-eight patients $(38 \%)$ were in the gray zone.

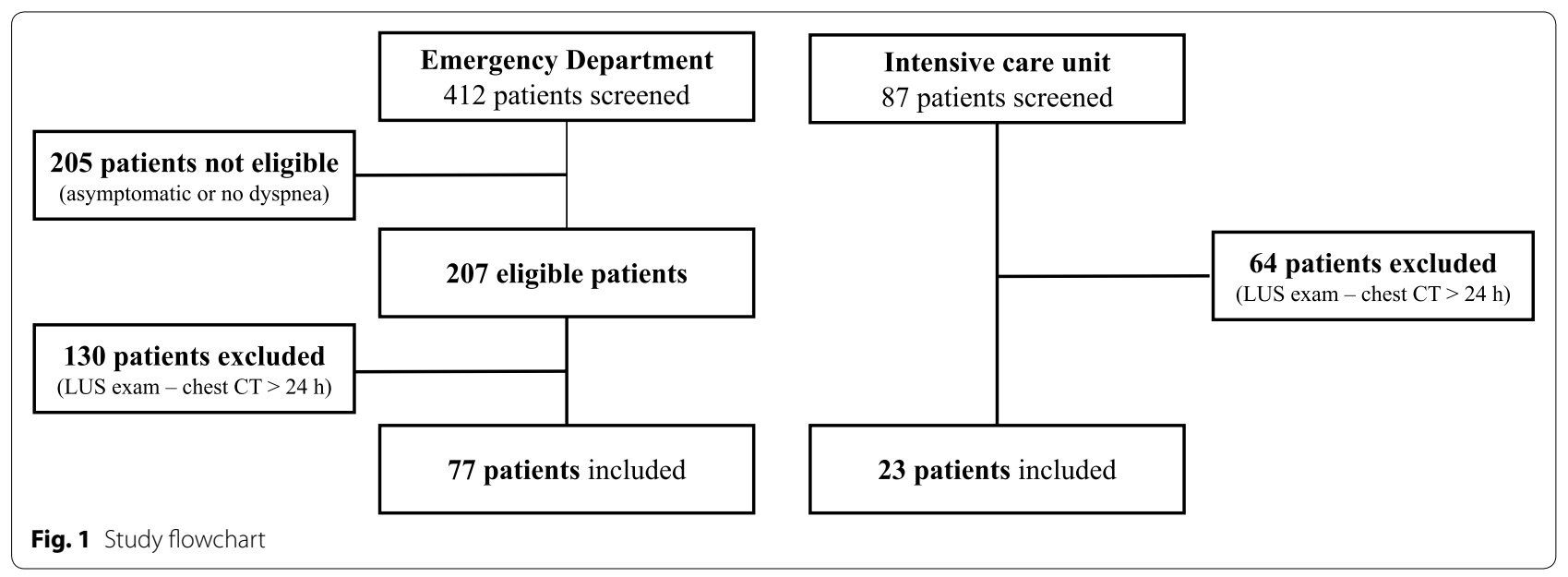


Table 1 Baseline clinical data

\begin{tabular}{|c|c|c|c|c|}
\hline Patient information & $\begin{array}{l}\text { All } \\
n=100\end{array}$ & $\begin{array}{l}\text { ED } \\
n=77\end{array}$ & $\begin{array}{l}\mathrm{ICU} \\
n=23\end{array}$ & $P$ value \\
\hline \multicolumn{5}{|l|}{ Characteristics } \\
\hline Age, median (IQR), years & $61(54-77)$ & $60(51-78)$ & $69(55-73)$ & 0.49 \\
\hline \multicolumn{5}{|l|}{ Sex } \\
\hline Men (\%) & $65(65)$ & $45(58)$ & $20(87)$ & \multirow[t]{2}{*}{0.01} \\
\hline Women (\%) & $35(35)$ & $32(42)$ & $3(13)$ & \\
\hline $\mathrm{BMI}>30$ kg/m (\%) & $17(17)$ & $11(14)$ & $6(26)$ & 0.21 \\
\hline \multicolumn{5}{|l|}{ Co-morbidities } \\
\hline Hypertension (\%) & $24(24)$ & $12(16)$ & $12(52)$ & 0.0003 \\
\hline Coronary disease (\%) & $11(11)$ & $5(7)$ & $6(26)$ & 0.02 \\
\hline Heart failure (\%) & $16(16)$ & $13(17)$ & $3(13)$ & 0.66 \\
\hline COPD (\%) & $10(10)$ & $8(10)$ & $2(9)$ & 0.81 \\
\hline Chronic kidney disease (\%) & $2(2)$ & $1(1)$ & $1(4)$ & 0.41 \\
\hline Liver disease (\%) & $1(1)$ & 0 & $1(4)$ & 0.23 \\
\hline Diabetes (\%) & $16(16)$ & $7(9)$ & $9(39)$ & 0.002 \\
\hline Immunodepression (\%) & $1(1)$ & $1(1)$ & 0 & 0.58 \\
\hline Cancer (\%) & $7(7)$ & $4(5)$ & $3(13)$ & 0.2 \\
\hline \multicolumn{5}{|l|}{ Clinical features } \\
\hline MAP, median (IQR), mmHg & $90(81-99)$ & $92(83-100)$ & $84(75-98)$ & 0.16 \\
\hline Heart rate, median (IQR), bpm & $91(82-104)$ & $91(82-104)$ & $91(82-105)$ & 0.82 \\
\hline Respiratory rate, median (IQR), bpm² & $23(18-28)$ & $22(18-28)$ & $26(20-30)$ & 0.20 \\
\hline $\mathrm{SpO}_{2}$, median (IQR), \% & $95(93-97)$ & $96(93-98)$ & $93(91-94)$ & 0.0001 \\
\hline Oxygen rate flow, median (IQR), L/min & $2(0-6)$ & $0(0-4)$ & $9(6-30)$ & 0.002 \\
\hline Mechanical ventilation, n (\%) & $7(7)$ & 0 & $7(30)$ & $<0.0001$ \\
\hline $\mathrm{PaO}_{2}$, median $(\mathrm{IQR}), \mathrm{mmHg}$ & $76(66-88)$ & $77(65-90)$ & $76(67-82)$ & 0.66 \\
\hline $\mathrm{SpO}_{2} / \mathrm{FiO}_{2}$ ratio, median (IQR) & $335(233-452)$ & $428(292-457)$ & $184(133-244)$ & $<0.0001$ \\
\hline $\begin{array}{l}\text { Delay between ultrasound exam and chest CT } \\
\text { scan, median (IQR), hours }\end{array}$ & $4(3-7)$ & $4(3-6)$ & $3(1-10)$ & 0.5 \\
\hline
\end{tabular}

Data are expressed as $n$ (\%) of participants, unless otherwise indicated

$\mathrm{BMI}$ body mass index; $\mathrm{COPD}$ chronic obstructive pulmonary disease; $\mathrm{MAP}$ mean arterial pressure; $\mathrm{SpO}_{2}$ pulse oximetry; $\mathrm{PaO}_{2}$ arterial oxygen partial; FiO fractional inspired oxygen; IQR interquartile range; $C T$ computed tomography, $E D$ emergency department; ICU intensive care unit

1 bpm: beats per minute

2 bpm: breath per minute

\section{Secondary outcomes}

The Se, Sp, PPV, NPV and DA of LUS for the chest CT scan diagnosis of alveolar consolidation, interstitial syndrome, pneumothorax, pleural effusion and pleural irregularity are reported in ESM Table S1. LUS score was associated with clinical status at the time of examination. The LUS score was significantly higher in the mechanically ventilated patients $(28 \pm 5$ vs. $14 \pm 8$; $p<0.0001)$. All the mechanically ventilated patients had an LUS score $>19$. The ROC curve of LUS for mechanical ventilation was 0.92 . The LUS score was significantly higher in the patients with a $\mathrm{SpO}_{2} / \mathrm{FiO}_{2}$ ratio $<357$ $(19 \pm 8$ vs. $11 \pm 8 ; p<0.0001)$.

\section{Discussion}

Our results show that the severity of SARS-CoV-2 pneumonia assessed by LUS is highly associated with severity as assessed by chest CT scan. Thus, LUS could replace chest CT scan for the initial assessment of lung involvement in most of confirmed symptomatic SARS-CoV-2 patients.

A chest CT scan is the gold standard to assess the severity of pneumonia in patients with SARS-CoV-2 [25]. An international consensus statement concluded that 'In a resource-constrained environment, imaging is indicated for medical triage of patients with suspected SARS-CoV-2 who present with moderate-severe 
Table 2 CT and LUS findings

\begin{tabular}{|c|c|c|c|}
\hline Findings & CT $n=100$ & LUS $n=100$ & $P$ value \\
\hline \multicolumn{4}{|l|}{ Interstitial syndrome } \\
\hline Absent & $8(8)$ & $4(4)$ & \multirow[t]{3}{*}{0.134} \\
\hline Unilateral & $5(5)$ & $11(11)$ & \\
\hline Bilateral & $87(87)$ & $85(85)$ & \\
\hline \multicolumn{4}{|l|}{ Consolidation } \\
\hline Absent & $48(48)$ & $68(68)$ & \multirow[t]{3}{*}{0.002} \\
\hline Unilateral & $16(16)$ & $17(17)$ & \\
\hline Bilateral & $36(36)$ & $15(15)$ & \\
\hline \multicolumn{4}{|l|}{ Pleural effusion } \\
\hline Absent & $89(89)$ & $94(94)$ & \multirow[t]{3}{*}{0.122} \\
\hline Unilateral & $7(7)$ & $6(6)$ & \\
\hline Bilateral & $4(4)$ & 0 & \\
\hline Pneumothorax & 0 & 0 & - \\
\hline Pleural irregularity & $15(15)$ & $32(32)$ & 0.005 \\
\hline
\end{tabular}

clinical features and a high pre-test probability of disease' [26]. However, there are risks and logistical limitations to the use of CT. The transfer of unwell patients risks adverse events $[5,6]$, there is increased potential exposure to the virus of all the healthcare providers involved in the transfer, and there may be a lack of scanning capacity if there is a high number of patients presenting. Unlike CT scan, LUS has the advantages that it is available at the point of care, it may be performed by suitably skilled physicians in the ED or ICU, and there is negligible cost to each individual examination [27]. The same trends were previously reported for the H1N1 pandemic [11], and our results confirm similar utility of LUS to assess the severity of SARSCoV-2 pneumonia. In addition, due to the high incidence of thromboembolic events [28, 29], LUS should be incorporated into multiorgan ultrasound assessment to detect both venous thrombosis $[30,31]$ and signs of acute right heart failure [32].

The discrepancy between LUS and CT scan results lies in the middle region, or gray zone, of LUS scores. From these results, we suggest that chest CT scan would not be required if an initial LUS examination found a score $<13$ (mild disease) or $>23$ (severe disease), but only in the $38 \%$ of cases with an intermediate score between 13 and 23. Furthermore, LUS exams were not performed exclusively by expert operators, and it might be assumed that improving the level of expertise would have improved the accuracy. In contrast, chest CT scans were all interpreted by senior radiologists with a high level of expertise.

LUS scores differed according to clinical status of our highly select population. Patients in the ICU had a higher $\mathrm{SpO}_{2} / \mathrm{FiO}_{2}$ ratio $\geq 357$. Furthermore, all the patients having mechanical ventilation had an LUS score $>19$.

Our study has several limitations. First, we included a convenience sample of patients with dyspnoea and confirmed PCR, which represents only a small percentage of the many cases of pneumonia observed during the pandemic. The chest CT scan and LUS were not performed simultaneously, but the median delay between chest CT scan and LUS was four hours. As previously described [20], we used a modified LUS technique that did not scan the paravertebral areas. If this introduced any alteration in the diagnostic accuracy, it would be expected to have reduced identification of pulmonary consolidation, and hence the assessed degree of severity.

We wished to ensure that patients who were studied had both significant and proven SARS-CoV-2 infection. The delay in getting test results has led to situations where $\mathrm{CT}$ scan is being used as the primary investigation to diagnose and triage patients. As a positive PCR test was one of the inclusion criteria for our analysis, we cannot comment on whether our findings would apply to patients who had an expedited scan on this basis.

We suggest that further research should assess the generalisability of the low and high thresholds suggested above for replacement of CT scan by LUS as a tool to assess pneumonia severity, and the use of LUS as a predictor of the clinical course and a guide to ongoing management [33, 34].

Table 3 Correlation: LUS score according to chest CT scan severity

\begin{tabular}{|c|c|c|c|c|c|c|c|}
\hline \multirow[t]{2}{*}{ Chest CT scan severity } & \multirow[t]{2}{*}{ Patients } & \multirow{2}{*}{$\begin{array}{l}\text { LUS score } \\
\text { mean } \pm S D\end{array}$} & \multicolumn{2}{|c|}{$95 \%$ Cl for Mean } & \multirow{2}{*}{$\begin{array}{l}\text { LUS score } \\
\text { min }\end{array}$} & \multirow[t]{2}{*}{ LUS score max } & \multirow[t]{2}{*}{$P$ value } \\
\hline & & & Lower bound & Upper bound & & & \\
\hline Minimal damage & 18 & $8 \pm 7$ & 4 & 11 & 0 & 22 & $<0.0001$ \\
\hline Moderate damage & 43 & $14 \pm 8$ & 11 & 16 & 0 & 30 & \\
\hline Severe damage & 39 & $20 \pm 8$ & 18 & 23 & 5 & 34 & \\
\hline
\end{tabular}

CT computed tomography; LUS lung ultrasound; $C$ : confidence interval, SD standard deviation 


\section{a ROC curve of LUS score versus chest CT scan}

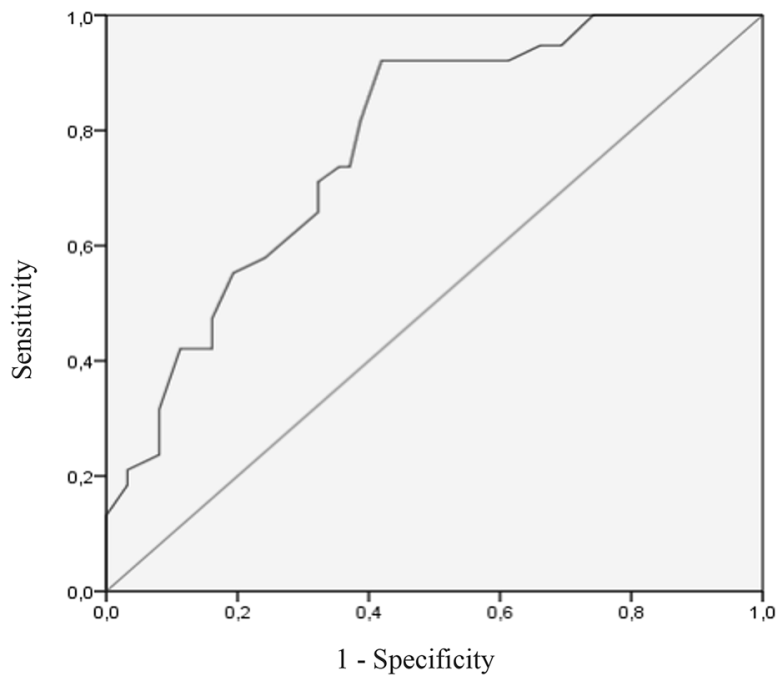

\section{b Grey zone of LUS score versus chest CT scan}

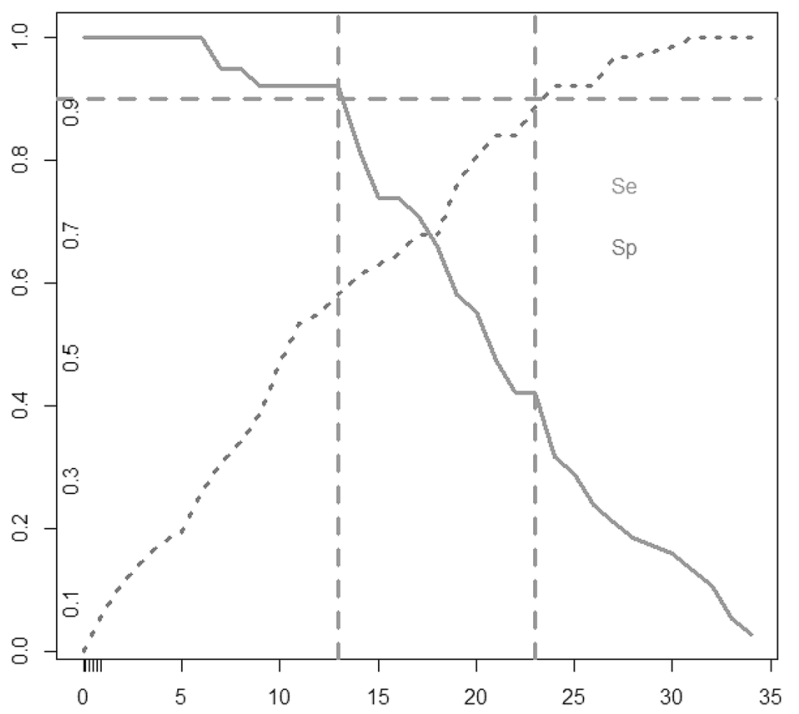

Fig. 2 Diagnostic accuracy of LUS score for the detection of severe SARS-CoV-2 pneumonia assessed by chest CT scan. a ROC curve of LUS score versus chest CT scan. AUC $=0.78$ (Cl 95\% 0.68-0.87; $p<0.0001)$, (b) Gray zone of LUS score versus chest CT scan. Solid line: Se (Sensitivity). Dotted line: $\mathrm{Sp}$ (Specificity)

\section{Conclusion}

In patients with proven SARS-CoV-2 pneumonia, LUS score is associated with severity as assessed by chest CT scan and clinical features.

\section{Electronic supplementary material}

The online version of this article (https://doi.org/10.1007/s00134-020-06186-0) contains supplementary material, which is available to authorized users.

\section{Abbreviations}

AUC: Area under curve; COVID-19: Coronavirus disease 2019; Cl: Confidence interval; CT: Computed tomography; DA: Diagnostic accuracy; ED: Emergency department; ESM: Electronical Supplemental Material; $\mathrm{FiO}_{2}$ : Fractional inspired oxygen; ICU: Intensive care unit; LUS: Lung ultrasound; NPV: Negative predictive value; $\mathrm{PaO}_{2}$ : Arterial oxygen partial; PCR: Polymerase chain reaction; PPV: Positive predictive value; ROC: Receiver operating characteristic; SARS-CoV-2: Severe acute respiratory syndrome coronavirus 2; Se: Sensitivity; Sp: Specificity; $\mathrm{SpO}_{2}$ : Pulse oximetry.

\section{Author details}

${ }^{1}$ Department of Anesthesiology and Intensive Care Medicine, Hôpital Nord, Assistance Publique Hôpitaux de Marseille, Aix Marseille University, 13015 Marseille, France. ${ }^{2}$ Center for Cardiovascular and Nutrition Research (C2VN), INRA, Aix Marseille Université, INSERM, 13005 Marseille, France. ${ }^{3}$ Department of Emergency Medicine, Timone University Hospital, Marseille, France. ${ }^{4}$ Centre D'Etudes Et de Recherches Sur Les Services de Santé Et Qualité, Faculté de Médecine, Aix-Marseille Université, 13005 Marseille, France. ${ }^{5}$ Department of Anesthesiology and Intensive Care MedicineUniversity Hospital of Besancon, University of Franche-Comte, 2. EA3920, Besancon, France. ${ }^{6}$ Centre Hospitalier Lyon-Sud, Hospices Civils de Lyon, Service de Réanimation, 69310 Pierre-Bénite, France. ${ }^{7}$ Service Anesthésie Réanimation, Groupement Hospitalier Est, Hospices Civils de Lyon, Lyon, France. ${ }^{8}$ Lyon1, Université Claude Bernard, Villeurbanne, France. ${ }^{9}$ VetAgro Sup, Pulmonary and Cardiovascular Aggresion in Sepsis, UPSP 2016.A101, Université de Lyon, Campus Vétérinaire de Lyon, 69280 Marcy l'Étoile, France. ${ }^{10}$ Assistance
Publique Hôpitaux de Marseille, Hôpital Nord, Aix Marseille University, Service d'Imagerie Médicale, 13015 Marseille, France.

\section{Acknowledgements}

We wish to thank Mike Kinsella for his help in editing the manuscript. We thank Eloïse Cercueil for her help in patient recruitment.

\section{Author contributions}

The corresponding author (LZ) attests that all listed authors meet the authorship criteria and that no others meeting the criteria have been omitted. LZ: Contributions: This author helped design and conduct the study, analyze the data and write the manuscript. Thibaut Markarian: Contributions: This author helped design and conduct the study, analyze the data and write the manuscript. AL: Contributions: This author helped conduct the study, analyze the data and write the manuscript. CT: Contributions: This author helped conduct the study. NM: Contributions: This author helped conduct the study. MB: Contributions: This author helped analyze the data and write the manuscript. KB: Contributions: This author helped analyze the data and write the manuscript. GB: Contributions: This author helped conduct the study and write the manuscript. GM: Contributions: This author helped conduct the study. GD: Contributions: This author helped conduct the study. LB: Contributions: This author helped write the manuscript. PM: Contributions: This author helped conduct the study. BA: Contributions: This author helped conduct the study. KC: Contributions: This author helped conduct the study, analyze the data and write the manuscript. MB: Contributions: This author helped conduct the study, analyze the data and write the manuscript. ML: Contributions: This author helped design the study and write the manuscript.

\section{Compliance with ethical standards}

\section{Conflicts of interest}

LZ and TM received fees for teaching ultrasound to GE healthcare customers.

\section{Publisher's Note}

Springer Nature remains neutral with regard to jurisdictional claims in published maps and institutional affiliations. 
Received: 28 April 2020 Accepted: 16 July 2020

Published online: 29 July 2020

\section{References}

1. Li Y, Xia L (2020) Coronavirus disease 2019 (COVID-19): role of chest CT in diagnosis and management. AJR Am J Roentgenol 1:7. https://doi. org/10.2214/AJR.20.22954

2. AiT, Yang Z, Hou H et al (2020) Correlation of chest CT and RT-PCR testing in coronavirus disease 2019 (COVID-19) in China: a report of 1014 cases. Radiology 2:642. https://doi.org/10.1148/radiol.2020200642

3. Wu J, Wu X, Zeng W et al (2020) Chest CT findings in patients with coronavirus disease 2019 and its relationship with clinical features. Invest Radiol 55:257-261. https://doi.org/10.1097/RLI.0000000000000670

4. Mahdjoub E, Mohammad W, Lefevre T et al (2020) Admission chest CT score predicts 5-day outcome in patients with COVID-19. Intensive Care Med. https://doi.org/10.1007/s00134-020-06118-y

5. Aliaga M, Forel J-M, De Bourmont S et al (2015) Diagnostic yield and safety of CT scans in ICU. Intensive Care Med 41:436-443. https://doi. org/10.1007/s00134-014-3592-1

6. Jia L, Wang H, Gao Y et al (2016) High incidence of adverse events during intra-hospital transport of critically ill patients and new related risk factors: a prospective, multicenter study in China. Crit Care 20:12. https://doi. org/10.1186/s13054-016-1183-y

7. Hope MD, Raptis CA, Shah A et al (2020) A role for CT in COVID-19? What data really tell us so far. Lancet 395:1189-1190. https://doi.org/10.1016/ S0140-6736(20)30728-5

8. Lichtenstein D, Goldstein I, Mourgeon E et al (2004) Comparative diagnostic performances of auscultation, chest radiography, and lung ultrasonography in acute respiratory distress syndrome. Anesthesiology 100:9-15. https://doi.org/10.1097/00000542-200401000-00006

9. Xirouchaki N, Magkanas E, Vaporidi K et al (2011) Lung ultrasound in critically ill patients: comparison with bedside chest radiography. Intensive Care Med 37:1488-1493. https://doi.org/10.1007/s00134-011-2317-y

10. Staub L, Mazzali Biscaro RR, Kaszubowski E, Maurici R (2019) Lung ultrasound for the emergency diagnosis of pneumonia, acute heart failure, and exacerbations of chronic obstructive pulmonary disease/asthma in adults: a systematic review and meta-analysis. J Emerg Med 56:53-69. https://doi.org/10.1016/j.jemermed.2018.09.009

11. Testa A, Soldati G, Copetti R et al (2012) Early recognition of the 2009 pandemic influenza A (H1N1) pneumonia by chest ultrasound. Crit Care 16:R30. https://doi.org/10.1186/cc11201

12. Buonsenso D, Pata D, Chiaretti A (2020) COVID-19 outbreak: less stethoscope, more ultrasound. Lancet Respir Med. https://doi.org/10.1016/ S2213-2600(20)30120-X

13. Lomoro P, Verde F, Zerboni F et al (2020) COVID-19 pneumonia manifestations at the admission on chest ultrasound, radiographs, and CT: singlecenter study and comprehensive radiologic literature review. Eur J Radiol Open 7:100231. https://doi.org/10.1016/j.ejro.2020.100231

14. Peng Q-Y, Wang X-T, Zhang L-N, Chinese Critical Care Ultrasound Study Group (CCUSG) (2020) Findings of lung ultrasonography of novel corona virus pneumonia during the 2019-2020 epidemic. Intensive Care Med. https://doi.org/10.1007/s00134-020-05996-6

15. Duclos G, Lopez A, Leone M, Zieleskiewicz L (2020) “No dose” lung ultrasound correlation with "low dose" CT scan for early diagnosis of SARS-cov-2 pneumonia. Intensive Care Med. https://doi.org/10.1007/ s00134-020-06058-7

16. Toulouse E, Masseguin C, Lafont B et al (2018) French legal approach to clinical research. Anaesth Crit Care Pain Med 37:607-614. https://doi. org/10.1016/j.accpm.2018.10.013

17. Malas O, Cağlayan B, Fidan A et al (2003) Cardiac or pulmonary dyspnea in patients admitted to the emergency department. Respir Med 97:1277-1281. https://doi.org/10.1016/j.rmed.2003.07.002
18. Frat J-P, Thille AW, Mercat A et al (2015) High-flow oxygen through nasal cannula in acute hypoxemic respiratory failure. N Engl J Med 372:21852196. https://doi.org/10.1056/nejmoa1503326

19. Pandharipande PP, Shintani AK, Hagerman HE et al (2009) Derivation and validation of spo2/fio2 ratio to impute for pao2/fio2 ratio in the respiratory component of the Sequential Organ Failure Assessment (SOFA) Score. Crit Care Med 37:1317-1321. https://doi.org/10.1097/CCM.0b013 e31819cefa9

20. Mojoli F, Bouhemad B, Mongodi S, Lichtenstein D (2018) Lung ultrasound for critically III patients. Am J Respir Crit Care Med 199:701-714. https:// doi.org/10.1164/rccm.201802-0236Cl

21. Arbelot C, Neto FLD, Gao Y et al (2020) Lung ultrasound in emergency and critically III patientsnumber of supervised exams to reach basic competence. Anesthesiology 132:899-907. https://doi.org/10.1097/ ALN.0000000000003096

22. Volpicelli G, Elbarbary M, Blaivas M et al (2012) International evidencebased recommendations for point-of-care lung ultrasound. Intensive Care Med 38:577-591. https://doi.org/10.1007/s00134-012-2513-4

23. Ray P, Le Manach Y, Riou B, Houle TT (2010) Statistical evaluation of a biomarker. Anesthesiology 112:1023-1040. https://doi.org/10.1097/ ALN.0b013e3181d47604

24. Coste J, Pouchot J (2003) A grey zone for quantitative diagnostic and screening tests. Int J Epidemiol 32:304-313. https://doi.org/10.1093/ije/ dyg054

25. Zhao W, Zhong Z, Xie X et al (2020) Relation between chest CT Findings and clinical conditions of coronavirus disease (COVID-19) pneumonia: a multicenter study. Am J Roentgenol 214:1072-1077. https://doi. org/10.2214/AJR.20.22976

26. Rubin GD, Ryerson CJ, Haramati LB et al (2020) The role of chest imaging in patient management during the COVID-19 pandemic: a multinational consensus statement from the fleischner society. Chest. https://doi. org/10.1016/j.chest.2020.04.003

27. Mayo PH, Copetti R, Feller-Kopman D et al (2019) Thoracic ultrasonography: a narrative review. Intensive Care Med 45:1200-1211. https://doi. org/10.1007/s00134-019-05725-8

28. Helms J, Tacquard C, Severac F et al (2020) High risk of thrombosis in patients with severe SARS-cov-2 infection: a multicenter prospective cohort study. Intensive Care Med 46:1089-1098. https://doi.org/10.1007/ s00134-020-06062-x

29. Tavazzi G, Civardi L, Caneva L et al (2020) Thrombotic events in SARSCov-2 patients: an urgent call for ultrasound screening. Intensive Care Med. https://doi.org/10.1007/s00134-020-06040-3

30. Koenig S, Chandra S, Alaverdian A et al (2014) Ultrasound assessment of pulmonary embolism in patients receiving CT pulmonary angiography. Chest 145:818-823. https://doi.org/10.1378/chest.13-0797

31. Nazerian P, Volpicelli G, Gigli C et al (2017) Diagnostic performance of wells score combined with point-of-care lung and venous ultrasound in suspected pulmonary embolism. Acad Emerg Med 24:270-280. https:// doi.org/10.1111/acem.13130

32. Creel-Bulos C, Hockstein M, Amin N et al (2020) Acute cor pulmonale in critically III patients with Covid-19. N Engl J Med 2:e70. https://doi. org/10.1056/nejmc2010459

33. Volpicelli G, Lamorte A, Villén T (2020) What's new in lung ultrasound during the COVID-19 pandemic. Intensive Care Med. https://doi.org/10.1007/ s00134-020-06048-9

34. Markarian T, Zieleskiewicz L, Perrin G et al (2019) A lung ultrasound score for early triage of elderly patients with acute dyspnea. CJEM 21:399-405. https://doi.org/10.1017/cem.2018.483 